\title{
Neuropsychiatric profile of a male child with tuberous sclerosis complex.
}

\author{
Eman A Zaky*, Rana AA Mahmoud \\ Child Psychiatry Unit, Department of Pediatrics, Faculty of Medicine, Ain Shams University, Egypt
}

\begin{abstract}
Tuberous sclerosis complex is a disorder that affects many systems of the body including the central nervous system, the cardiovascular system, the skin, the kidneys, the lungs, and the retina. Much neuropsychiatric comorbidity is recorded in individuals suffering from the disease. This is a report of a 12 year old boy suffering from tuberous sclerosis complex with chief complaints of generalized tonic-clonic fits that have started at the age of 3 years, cognitive impairment that was recognized when he was a toddler and disturbing behavioral problems over the last five to six years. His behavioral problems included hyperactivity, rage attacks, stubbornness, and physical and verbal aggression against his family members in addition to frequent exhibitionism and micturition in public. Furthermore, our patient experiences episodes of binge eating with purging behavior and vocal tics (grunting and throat clearing sounds). As the management of such behavior problems is a point of debate among concerned professionals and they are typically agonizing, handicapping, and disturbing for the patients and their caregivers, we reported this case to attract the attention of the primary care physicians and general pediatricians not to undermine their significant impact in comparison to the other serious physical problems such children and adolescents are usually suffering from.
\end{abstract}

Keywords: Tuberous sclerosis, Tuberous sclerosis complex, Challenging behavior, Intellectual disability, Hamartin, Tuberin, Autosomal dominant disorder, TSC1, TSC2.

Accepted on February 07, 2018

\section{Introduction}

Tuberous sclerosis complex (TSC) is a genetic disorder that is could be sporadic or inherited. If it is inherited it will follow the autosomal dominant pattern of inheritance. The responsible mutations for its occurrence involve two genes: TSC1 (9q34.3) which codes for hamartin and TSC2 (16p13.3) which codes for tuberin [1].

Despite the fact that TSC affects many organs, dermatological, neurological, and or renal involvements are by far the most common [2]. Skin manifestations are striking during childhood while renal affection is recorded in about $50 \%$ to $80 \%$ of cases and it is most commonly in the form of multiple bilateral renal angiomyolipomas that is considered a potentially lethal lesion as it can lead to serious spontaneous hemorrhage [3].

Neuropsychiatric disorders are striking among patients with TSC and represent a therapeutic challenge to concerned professionals. They include convulsions which affect the majority of cases and they could be focal or generalized developmental delay, intellectual disabilities, autism spectrum disorder, hyperactivity and aggressive behavior. Subependymal nodules are common and may be transformed into the potentially fatal subependymal giant cell astrocytoma with the development of progressive hydrocephalus [4,5].

We are reporting a 12 year old child with handicapping neurobehavioral manifestations of TSC.

\section{Case Report}

A twelve-years-old male child presented to the Child
Psychiatric Clinic, Children's Hospital, Ain Shams University, Egypt with chief complaints of generalized tonic-clonic fits that have started at the age of 3 years, cognitive impairment that was recognized when he was a toddler, and disturbing behavioral problems over the last five to six years. He is suffering from attacks of convulsions which are variable in their frequency and duration and he has not enjoyed a seizure free period since its onset.

The reported child is the $5^{\text {th }}$ born of non-consanguineous marriage that has been born at term by spontaneous vaginal delivery and the perinatal period passed uneventfully. $\mathrm{He}$ acquired motor milestones at their average time while cognitive milestones were significantly delayed. He was breast fed and fully immunized to schedule.

The mother sought medical advice many times because of her son's complaints mainly seizures for which he is maintained currently on sodium valproate, carbamazepine and lamotrigine. The child did not succeed to join any type of schooling because of the repeated failures to pass the admission interviews due to the significant cognitive impairment he is suffering from. $\mathrm{He}$ likes to play with younger children living nearby but sometimes they feel that he is odd because of his strange habit of touching and tasting dead animals and their remains.

Over the last five to six years, he stated to develop progressive behavioral problems like hyperactivity, rage attacks, stubbornness, and physical and verbal aggression against his family members that is usually evoked by refusal of his demands. He does not get along with his age counterparts 
and consequently, he has no friends. Frequent exhibitionism and micturition in public leads to marked embarrassment for the mother and his whole family. In addition, our patient experiences episodes of binge eating with purging behavior and vocal tics (grunting and throat clearing sounds). He has no obsessiveness, compulsiveness, or substance abuse.

Currently, the reported child has 2 sisters and a brother who were clinically healthy while he has a sib who passed away at the age of 13 years because of serious post-operative complications after tonsillectomy. There were no similar conditions or other mental disorders among family members. Figure 1 shows the family pedigree of the proband.

On examination; the patient had fair general condition with normal temperature, pulse, and respiratory rate. His weight was on the $90^{\text {th }}$ percentile while his height was on the $10^{\text {th }}$ and occipitofrontal circumference was on the $50^{\text {th }}$ percentile. $\mathrm{H}$ had no pallor, cyanosis, jaundice, edema or enlarged lymph nodes.

Facial angiofibromas were striking (Figure 2) while the scalp showed 3 firm non tender swellings of variable sizes (Figure 3 ). Also, scalp hair showed right temporo-parietal hypopigmented hair tuft (poliosis) (Figure 4). Acanthosis negricans with skin tags were detected at the back of his neck and axillae (Figure 5). A shagreen patch was found over the lower back (Figure 6) with four hypomelanotic macules; the largest of which was detected in the upper lateral part of his right thigh (Figure 7). Confetti skin lesions were evident in the right axilla (Figure 8) with no periungual or subungual fibromas.

Neurologically, he was fully conscious, alert, well oriented to person, time, and place. He is right handed. Mental status examination showed his cognitive impairment and according to the DSM5 diagnostic criteria [6], he was diagnosed to have

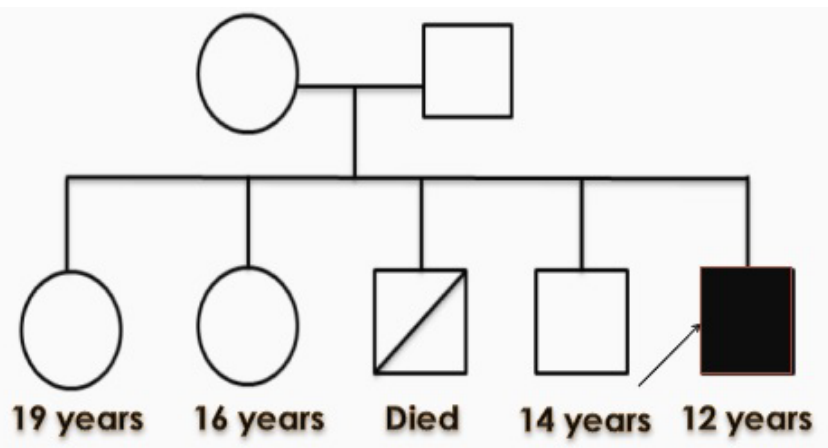

Figure 1. Shows the family pedigree of the reported case with TS.

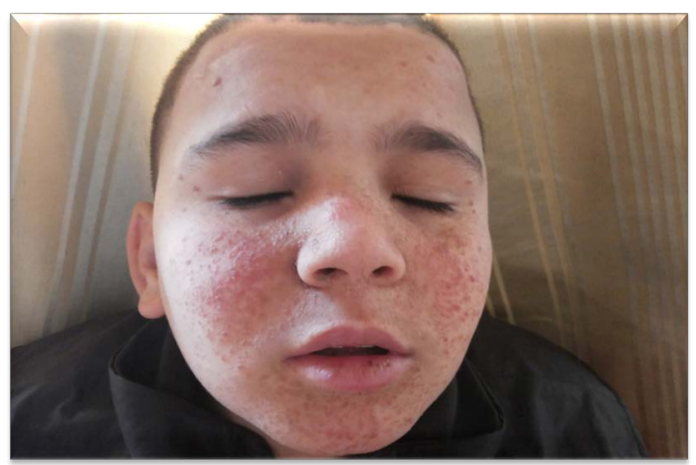

Figure 2. Facial malar angiofibromas sparing the upper lip. J Clin Psychiatry Cog Psychol 2018 Volume 2 Issue 1

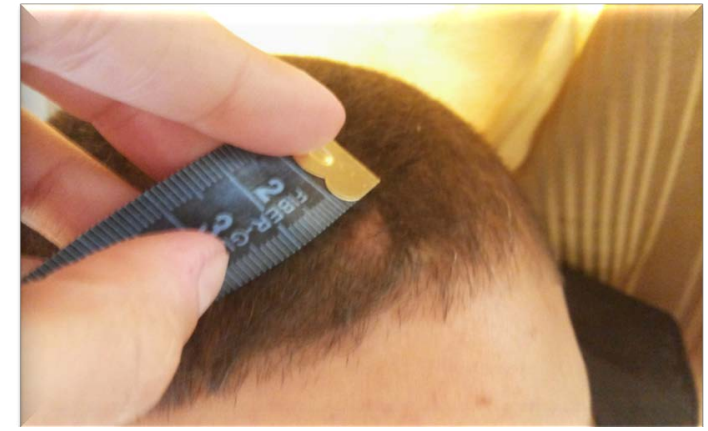

Figure 3. Scalp swelling (fibroma).

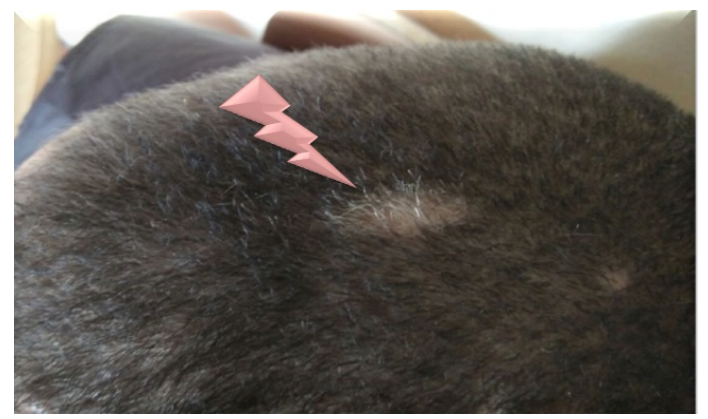

Figure 4. Poliosis of the scalp hair.

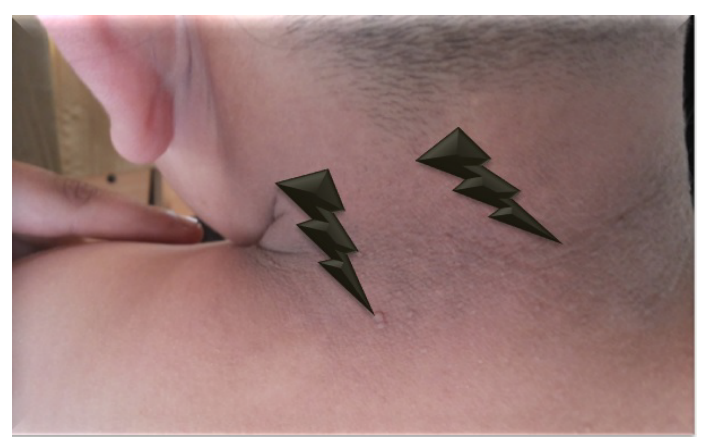

Figure 5. Acanthosis negricans and skin tags.

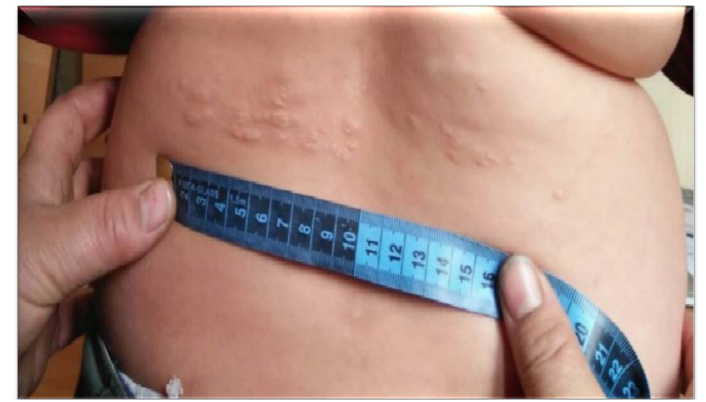

Figure 6. Left lumbar Shagreen patch.

binge-eating disorder, ADHD (according to his mental age), and other specified tic disorder that could be attributed to his TSC. His tics were only vocal (sounds of throat clearing and grunting) and of less than one year in duration. Cranial nerves were normal as well as his limbs muscle tone and power with neither deformities nor wasting. Abdominal examination showed mild non tender hepatomegaly. He was prepubertal (Tanner 1). The rest of his thorough physical examination revealed no other abnormalities.

The IQ was 45 fulfilling the DSM5 criteria of moderate intellectual disability [6]. $\mathrm{CBC}$, hepatic and renal functions 


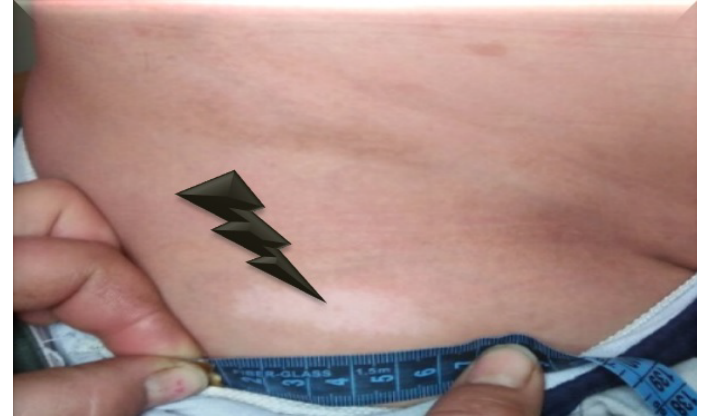

Figure 7. Right thigh ash leaf macule.

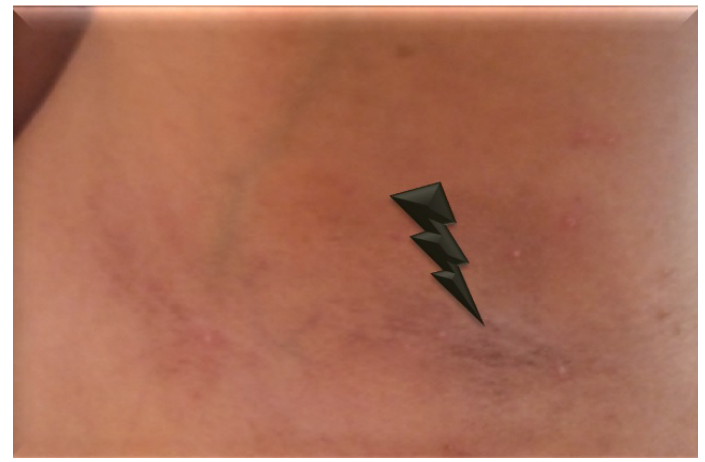

Figure 8. Right axilla confetti skin lesions.

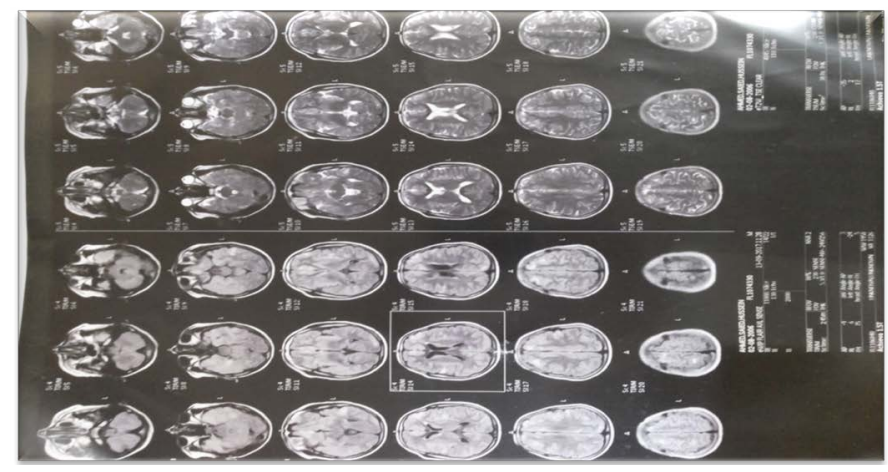

Figure 9. Brain MRI showing multiple cortical and subependymal tuberous lesions.

tests, ECG and fundus examination were normal while electroencephalogram (EEG) revealed focal epileptogenic discharge on the right temporal and occipital regions.

Imaging was done including pelvi-abdominal sonographic examination that showed many clear renal cysts with many well defined echogenic focal lesions because of renal angiomyolipomas. An intramural rhabdomyoma within the interventricular septum was detected by echocardiography. Brain MRI revealed many cortical and subependymal tubers with the largest one at the right foramen of Monro (Figure 8).

The patient started behavioral therapy and parents were advised what to do during the exacerbation of his behavioral problems. As a part of the management plan, the patient anticonvulsants' dosages were optimized. Lamotrigine improved his seizure control but he developed visual hallucinations; a form of occipital lobe hyperactivity, for which his medications were modified. Anticonvulsants might share in the exaggeration of his behavior problems. Hopefully, adjustment of his medications together with behavior therapy will help in controlling his 8 neuropsychiatric problems and improve his quality of life (Figure 9).

\section{Discussion}

Tuberous sclerosis complex is a multi-system disease with increased tendency for the development of benign neoplasms (hamartomas) in different organs like the brain, the retina, the lungs, the kidneys and the heart with significant cutaneous involvement [7].

The development of cortical tubers is responsible for the occurrence of many neuropsychiatric manifestations including convulsions, intellectual disability and behavior problems [8].

On the other hand, dermatological involvement is reported in the majority of TSC cases and they represent significant diagnostic clues. They may be in the form of hypopigmented macules, facial angiofibromas, Shagreen patch, non-traumatic ungula or periungual fibromas and confetti skin lesions [5]. It is worthy to mention that hypopigmented macules are reported in $90 \%$ of TSC patients and are detectable early in life before the development of other skin manifestations [9].

The currently reported case developed the diagnostic criteria of TSC early in life in the form of convulsions, developmental delay and a variety of skin lesions. Interestingly, he had a spectrum of behavioral problems that was very disturbing to his family members and others living nearby. As TSC is an autosomal dominant disorder and the reported case has no family history of similar conditions, his condition is attributed to a fresh mutation from unaffected parents like the majority of TSC cases [10].

TSC consensus revised criteria classified cases into definite, probable, or possible depending on the recorded major and minor features. A definitive case will have either 2 major or one major and two minor features while a probable case is diagnosed when the patient has one major and one minor feature. Lastly, possible case is suspected with one major or two or more minor features [11]. The currently reported case is considered a definitive one as he had many major features (facial angiofibromas, ash leaf macules, Shagreen patch, cortical and subependymal tubers, cardiac rhabdomyoma and renal angiomyolipoma) and two minor features (multiple renal cysts and Confetti skin lesions.

The management plan for our case was tailored according to the priorities of his disturbing manifestations; seizures and behavioral problems. His anticonvulsant medications are adjusted and closely monitored and behavior therapy with involvement of his family members was implemented to control his challenging behavior aiming at improving the chances to be accepted and included by his society and accordingly enjoying a better quality of life.

\section{Conclusion}

Cases with TSC must be thoroughly evaluated by multidisciplinary teams to detect the possible multi-system manifestations as early as possible and implement the needed early therapeutic interventions in a prompt way in order to improve their prognosis. Because behavioral manifestations 
of TSC patients are agonizing, handicapping, and disturbing for the patients and their caregivers, we reported this case to attract the attention of the primary care physicians and general pediatricians not to undermine their impact in comparison to the other serious physical problems such children and adolescents are suffering from. So, they need to be acknowledged and dealt with appropriately to improve the outcomes of the individualized management plans of such cases and their quality of life.

\section{References}

1. Au KS, Williams AT, Roach ES, et al. Genotype/phenotype correlation in 325 individuals referred for a diagnosis of tuberous sclerosis complex in the United States. Genet Med. 2007;9:88-100.

2. Curatolo P, Bombardieri R, Jozwiak S. Tuberous sclerosis. Lancet. 2008;372:657-68.

3. Schwartz RA, Fernández G, Kotulska K, et al. Tuberous sclerosis complex: Advances in diagnosis, genetics and management. J Am Acad Dermatol. 2007;57:189-202.

4. Goh S, Butler W, Thiele EA. Subependymal giant cell tumors in tuberous sclerosis complex. Neurology. 2004;63:1457-61.

5. Daroff RB, Fenichel GM, Jankovic J, et al. Bradley's
Neurology in Clinical Practice, $6^{\text {th }}$ edn. Philadelphia, PA. Elsevier. 2012;1508-14.

6. DSM 5 Diagnostic and Statistical Manual of Mental Disorders, $5^{\text {th }}$ edn. American Psychiatric Association. Arlington. 2013.

7. Barron RP, Kainulainen VT, Forrest CR, et al. (2002) Tuberous sclerosis clinicopathologic features and review of literature. J Craniomaxillofac Surg. 2002;30:361-6.

8. Curatolo P. Neurological manifestations of tuberous sclerosis complex. Child Nerv Syst. 1996;12:515-21.

9. Fitzpatrick TB, Szabo G, Hori Y, et al. White leaf-shaped macules, earliest visible sign of tuberous sclerosis. Arch Dermatol. 1968;98:1.

10. Crino PB, Nathanson KL, Henske EP. The tuberous sclerosis complex. N Engl J Med. 2006;355:1345-56.

11. Northrup H, Krueger. DA \& on the behalf of the International Tuberous Sclerosis Complex Consensus Group tuberous sclerosis complex diagnostic criteria Update: Recommendations of the 2012 International Tuberous Sclerosis Complex Consensus Conference. Pediatr Neurol. 2013;49:243-54.

\section{*Correspondence to:}

Prof. Eman Ahmed Zaky

$\mathrm{MD}, \mathrm{PhD}, \mathrm{DPP}$

Professor of General and Developmental and Behavioral Pediatrics and Clinical Genetics

Head of Child Psychiatry Unit

Department of Pediatrics

Faculty of Medicine

Ain Shams University

Egypt

Tel: 00202-01062978734

E-mail: emanzaky@med.asu.edu.eg 